\title{
Profiles of energetic muons in the atmosphere
}

\author{
Thomas K. Gaisser \\ Bartol Research Institute and Dept. of Physics and Astronomy \\ University of Delaware, Newark, DE, USA \\ Stef Verpoest \\ Dept. of Physics and Astronomy, University of Gent, B-9000 Gent, Belgium
}

\begin{abstract}
The production spectrum of high-energy muons as a function of depth in the atmosphere is relevant for understanding properties of event rates in deep detectors. For a given atmospheric profile, cascades of heavy nuclei develop at higher altitude than proton showers, giving rise to larger separation of muons at depth. For a given type of primary cosmic ray, seasonal variations in muon rates reflect the fact that higher temperatures correspond to lower densities and to a relative increase in the ratio of decay to re-interaction of the parent mesons. In this paper, we present a generalization of the Elbert formula that tracks meson decay to muons along the trajectory of the primary cosmic-ray nucleus. The convolution of the production spectrum with a changing atmospheric profile provides the dependence of event rates and sizes of muon bundles on temperature and primary mass. We consider applications to IceCube and also to multiple muon events in the compact underground detectors of MINOS and the NOvA Near Detector.
\end{abstract}

\section{Introduction}

The standard approach to seasonal variations uses formulas for the inclusive production spectrum of muons integrated over the primary spectrum [1]. The effective temperature $T_{\text {eff }}$ is obtained by weighting the production spectrum of muons as a function of atmospheric depth with the temperature

Email addresses: gaisser@udel .edu (Thomas K. Gaisser), stef.verpoest@ugent.be (Stef Verpoest) 
profile. The observed variation in the rate $I_{\mu}$ is then related to the variation in temperature by a correlation coefficient $\alpha_{T}$ according to

$$
\frac{\delta I_{\mu}}{\left\langle I_{\mu}\right\rangle}=\alpha_{T} \times \frac{\delta T_{\mathrm{eff}}}{\left\langle T_{\mathrm{eff}}\right\rangle},
$$

where the averages are typically taken for a full year. A general expression for the rate is

$$
I_{\mu}\left(E_{\mu, \min }, \theta\right)=\int \mathrm{d} X \int_{E_{\mu}, \min (\theta)} A_{\mathrm{eff}}\left(E_{\mu}, \theta\right) P\left(E_{\mu}, \theta, X\right) \mathrm{d} E_{\mu}
$$

where $E_{\mu, \min }(\theta)$ is the minimum energy for a muon to reach the detector from zenith angle $\theta{ }^{1}$ Here $X\left(\mathrm{~g} / \mathrm{cm}^{2}\right)$ is slant depth from the top of the atmosphere along a trajectory at zenith angle $\theta, A_{\text {eff }}$ is the projected effective area of the detector, and $P_{\mu}$ is the muon production spectrum. The full rate is obtained by integrating Eq. (2) over zenith angle.

In a large volume detector like IceCube $[2,3]$, the depth dependence of the response is accounted for with an energy-dependent effective area, and the production spectrum differential in energy must be used. For compact detectors like MINOS [4], however, the effective area is the physical area of the detector projected in the direction $\theta$ and averaged over azimuth. It depends only on $E_{\mu, \min }(\theta)$ and factors out of the integral in Eq. (2). In both cases, the formulas are "inclusive" in the sense that the primary spectrum has been integrated over to obtain the flux of muons per $\mathrm{m}^{2} \mathrm{~s} s \mathrm{~s}$.

In reality, underground detectors measure rates of events. This is especially the case for a large detector like IceCube, where high-energy events consist of large muon bundles from cores of air showers [5]. It is also the case for a tracking detector like MINOS when events are characterized by muon multiplicity and separation [6]. The goal of this paper is therefore to characterize the rate of events in terms of primary cosmic-ray energy and mass, while accounting for energy, multiplicity and height of origin of the muons.

The paper has three main sections. The first describes how muon production can be parameterized as a function of atmospheric depth and how the formulas integrated over depth relate to the Elbert formula [7, 8]. The

\footnotetext{
${ }^{1}$ The detectors we consider in this paper all have flat overburdens.
} 
following section considers coincident events in which a surface array provides an indication of the primary particle while the deep detector measures the properties of the muon bundle. The final section deals with underground measurements in which the weighted sum of all primaries is taken. The emphasis of this section is on seasonal variations of events in underground detectors and their dependence on muon multiplicity.

\section{Simulations and fitting parameters}

The formula originally proposed by Elbert [7, 8] as an approximation to the number of high-energy muons produced per primary cosmic ray, has been used to estimate properties of muon bundles in deep underground detectors $[9,10]$. A standard form $[11]$ is

$$
\left\langle N_{\mu}\left(>E_{\mu}, E_{0}, A, \theta\right)\right\rangle \approx A \times \frac{K}{E_{\mu} \cos \theta}\left(\frac{E_{0}}{A E_{\mu}}\right)^{\alpha_{1}}\left(1-\frac{A E_{\mu}}{E_{0}}\right)^{\alpha_{2}},
$$

where $A$ is the mass number of a primary nucleus of total energy $E_{0}$, and the values of the normalization constant $K$ and exponents $\alpha_{1}$ and $\alpha_{2}$ are included in the tables of parameters below. The scaling with $A E_{\mu} / E_{0}$ follows from the superposition approximation, in which incident nuclei are treated as $A$ independent nucleons each of energy $E_{0} / A$. In this paper we generalize the Elbert formula to obtain the distribution of slant depths over which the muons are produced. The integral of this distribution is the mean number of muons per shower, to be compared with Eq. (3). The basic idea is to interpret the derivative of the Gaisser-Hillas (G-H) function [12] as the rate of production of charged mesons per $\mathrm{d} X\left(\mathrm{~g} / \mathrm{cm}^{2}\right)$ along the trajectory of a primary cosmic ray and then multiply by appropriate factors to get the production spectrum for muons with energy $>E_{\mu}$,

$$
\begin{aligned}
\frac{\mathrm{d} N}{\mathrm{~d} X}\left(>E_{\mu}, E_{0}, A, \theta, X\right) & =N_{\max } \times \exp \left(\left(X_{\max }-X\right) / \lambda\right) \\
& \times\left(\frac{X-X_{0}}{X_{\max }-X_{0}}\right)^{\left(X_{\max }-X_{0}\right) / \lambda} \times \frac{X_{\max }-X}{\lambda\left(X-X_{0}\right)} \\
& \times F\left(E, E_{\mu}, \theta, X\right) \times \frac{1}{f E_{\mu} \cos \theta X} \times\left(1-\frac{A E_{\mu}}{E_{0}}\right)^{\alpha_{2}} .
\end{aligned}
$$

The first two lines on the right side of Eq. (4) are the derivative of the G-H formula. Because the application here is to hadronic cascades, the values of 


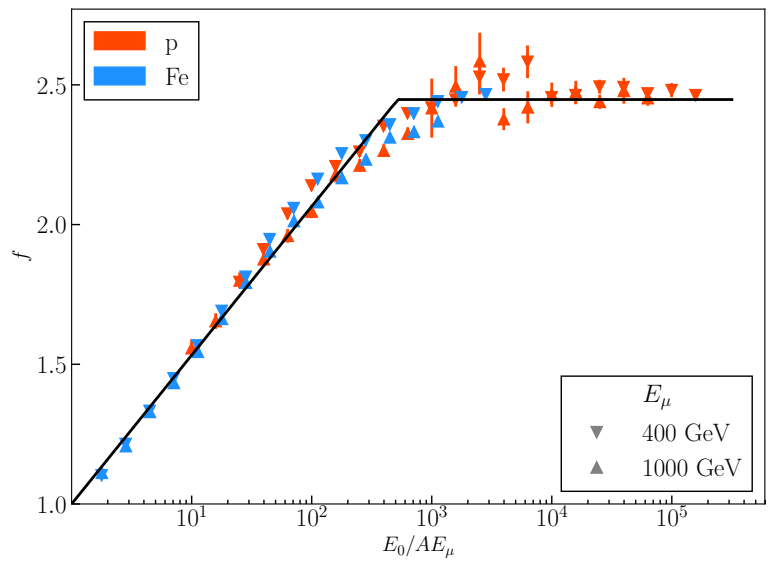

Figure 1: Value of the ratio $f$ of the minimum and mean (above minimum) muon energy in a shower. The black line is an approximate description of the values inferred from simulations of vertical proton and iron primary cosmic rays over a large range of primary energies and with two different minimum muon energies.

the parameters for number of particles at shower maximum $\left(N_{\max }\right)$, depth of shower maximum $\left(X_{\max }\right)$, starting depth $\left(X_{0}\right)$ and interaction scale $(\lambda)$ are numerically quite different from those of the original $\mathrm{G}-\mathrm{H}$ formula for air showers dominated by the electromagnetic cascade. In the last line, the first two factors give the probability of decay of a meson of energy $E$ to a muon of energy $E_{\mu}$ relative to the total rate of decay and re-interaction. The last factor is the threshold factor as in the Elbert formula. In the denominator of the decay factor, $E_{\mu}$ is replaced by $f E_{\mu}$ where the factor $f$ represents the ratio between the minimum muon energy and the mean energy of muons above this threshold. Its behaviour is inferred from simulations (described below) and can be seen in Fig. 1. There is an increase from 1 at threshold up to a value $f \approx 2.45$ at $E_{0} / A \gtrsim 10^{2.72} E_{\mu}$, after which it remains constant. The $\sec \theta$ factor reflects the increase of the muon flux with zenith angle, and the $1 / X$ dependence reflects the importance of high altitude (low density) for decay.

We consider two channels for muon production, $\pi^{ \pm} \rightarrow \mu+\nu_{\mu}(100 \%$ branching ratio) and decay of charged kaons (63.5\%). The decay fraction for charged pions with interaction length $\lambda_{\pi}$ is of the form

$$
\frac{1 / d_{\pi}}{1 / d_{\pi}+1 / \lambda_{\pi}},
$$


where

$$
\frac{1}{d_{\pi}}=\frac{\epsilon_{\pi}}{E_{\pi} \cos \theta X}
$$

and the pion critical energy is given by

$$
\epsilon_{\pi}=\frac{m_{\pi} c^{2}}{c \tau_{\pi}} \frac{R T}{M g} \approx 115 \mathrm{GeV} \times \frac{T}{220 \mathrm{~K}}
$$

where $m_{\pi}$ and $\tau_{\pi}$ are the mass and lifetime of the pion, $g$ is the gravitational constant, $R$ is the molar gas constant, $M=0.028964 \mathrm{~kg} / \mathrm{mol}$ for dry air and $T$ is the temperature. The critical energy for charged kaons is larger by a factor of 7.45 corresponding to its larger mass and shorter decay length. In pion decay, the muon carries an average energy of $E_{\mu}=r_{\pi} \times E_{\pi}$, with $r_{\pi} \approx$ 0.79. The corresponding factor for decay of charged kaons has $E_{\mu}=r_{K} \times E_{K}$ with $r_{K} \approx 0.52$. In Eq. (4) the common factor $1 / f E_{\mu} \cos \theta X$ is factored out so that

$$
F\left(E, E_{\mu}, \theta, X\right)=f_{\pi} \frac{r_{\pi} \epsilon_{\pi} \lambda_{\pi}}{1+\frac{r_{\pi} \epsilon_{\pi} \lambda_{\pi}}{f E_{\mu} \cos \theta X}}+f_{K} \frac{r_{K} \epsilon_{K} \lambda_{K}}{1+\frac{r_{K} \epsilon_{K} \lambda_{K}}{f E_{\mu} \cos \theta X}} .
$$

In this equation, $f_{\pi}=0.92$ and $f_{K}=0.08$ are the relative fractions of momentum carried by charged pions and charged kaons after accounting for the branching ratio 0.635 for kaon decay to muons. The numerical values are based on Fig. 5.2 of Ref. [11] where the momentum fraction carried by charged pions in p-air interactions is $Z_{N, \pi}(\gamma=1)=0.29$, and the fraction carried by charged kaons is 0.040 . So $f_{\pi}=0.29 /(0.29+0.635 \times 0.04)=0.92$, and $f_{K}=1-f_{\pi}=0.08$.

The muon production spectrum of Eq. (4) is fitted to simulations produced with CORSIKA [13] v7.7100 using Sibyll2.3c [14] as the high-energy interaction model and $\operatorname{UrQMD}[15,16]$ for interactions below $80 \mathrm{GeV}$, relevant for Section 4. An atmospheric profile corresponding to the average April South Pole atmosphere between 2007 and 2011 [17] was used. The fit of the formula to a muon production profile obtained from simulations has four free parameters, $N_{\max }, \lambda, X_{\max }$ and $X_{0}$, in the derivative of the G-H function. Fig. 2 shows examples of fitted longitudinal profiles of muon production over a range of primary energies for $300 \mathrm{GeV}$, normalized to the total number of muons produced for each primary energy and mass. Because the simulation includes production of muons from all channels, the contribution of kaons is included implicitly. The resulting optimal values of $N_{\max }, \lambda, X_{\max }$ and $X_{0}$ from repeating this procedure for a large number of muon and primary 


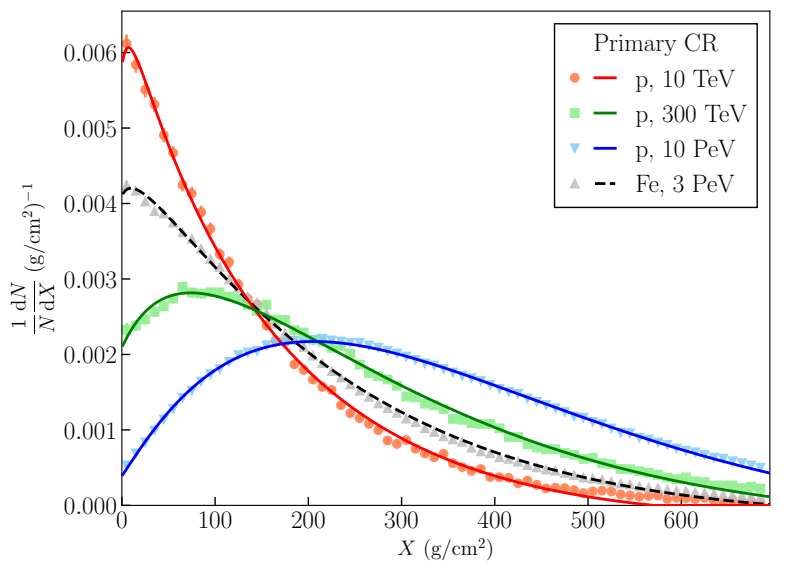

Figure 2: Differential muon production spectrum normalized to total $N_{\mu}$ for $E_{\mu} \geq 300 \mathrm{GeV}$ for different vertical primary cosmic rays. The markers are values obtained from CORSIKA simulations, the solid lines are the result of fitting Eq. (4) to these values.

cosmic ray energies can be seen in Fig. 3, where it becomes clear that they depend (in leading order) on $E_{0} / A E_{\mu}$. This behaviour is fit with the following functions,

$$
\begin{aligned}
N_{\max } & =c_{i} \times A \times\left(\frac{E_{0}}{A E_{\mu}}\right)^{p_{i}} \\
X_{\max }, \lambda, X_{0} & =a_{i}+b_{i} \times \log _{10}\left(\frac{E_{0}}{A E_{\mu}}\right),
\end{aligned}
$$

where $c_{i}, p_{i}, a_{i}$, and $b_{i}$ are defined for each function separately and have two regimes with a break at $R_{b}=\frac{E_{0}}{A E_{\mu}}=10^{q}$ and parameters $\left(a_{i}, b_{i}\right)$ with $i=1$ below the break and $i=2$ above. All parameters are listed in Table 1 for results based on simulations of vertical showers with $E_{\mu}>$ $300,400,500,700,1000 \mathrm{GeV}$. As noted in Ref. [18], the parameter $X_{0}$ is often negative in fitting individual showers and is to be considered simply as a parameter rather than the starting point of the cascade. For depths $X>X_{\max }$, Eq. (4) gives negative values, in which case we set it to zero.

As can already be seen in Fig. 3, the muon production profiles do not scale perfectly with the ratio of primary energy per nucleon and minimum muon energy, but show a remaining dependency on the latter. This is further illustrated in Fig. 4, where it becomes clear in the normalized production profiles 


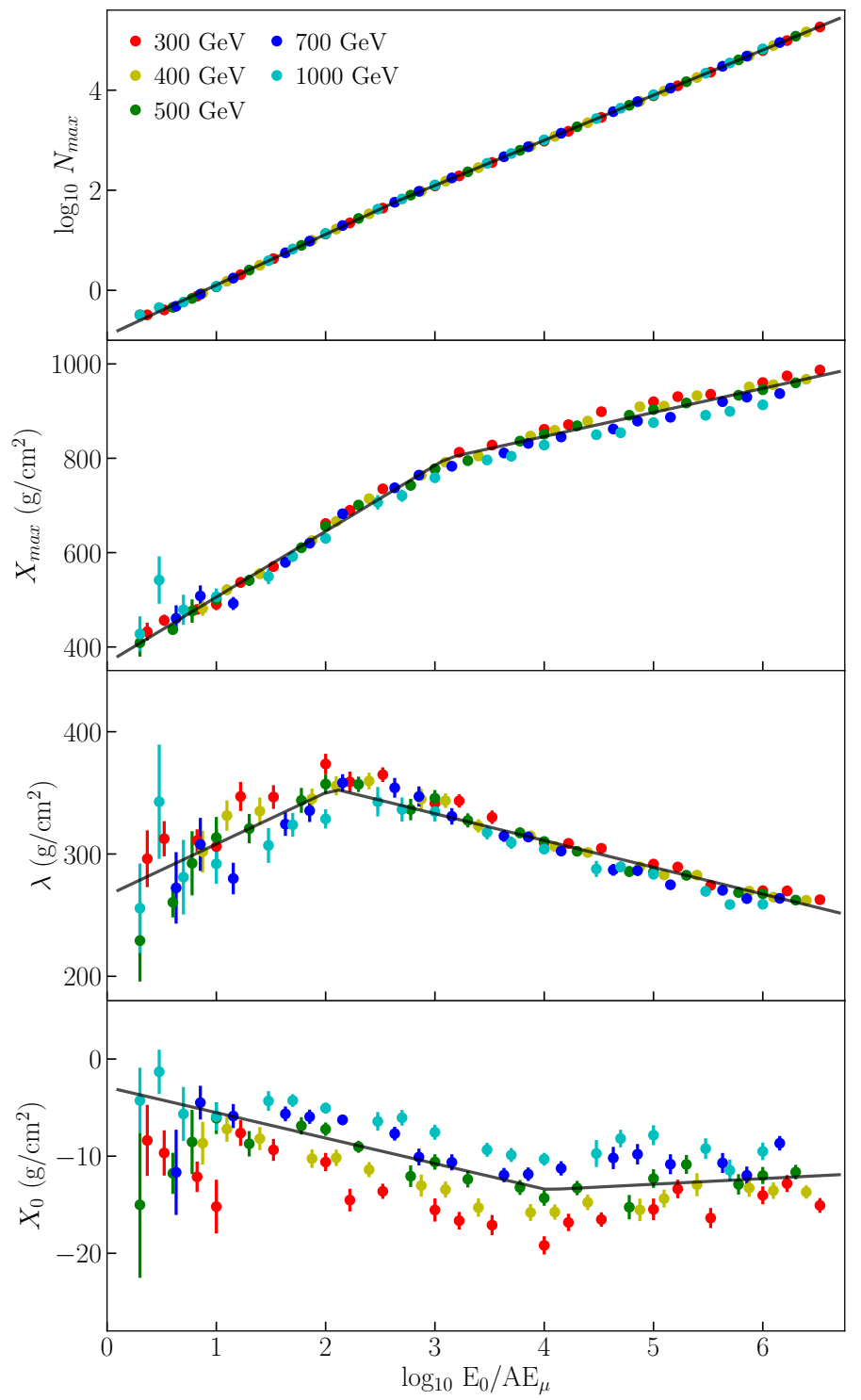

Figure 3: Optimal values of the four parameters in fits of the production spectrum Eq. (4) to simulations of vertical proton showers with different threshold muon energies over a large range of primary energies. The black line shows the approximate description given by Eq. (7) and Table 1. 
Table 1: Parameter values for Eq. (7) for $300 \mathrm{GeV} \lesssim E_{\mu} \lesssim 1 \mathrm{TeV}$.

\begin{tabular}{lr|r|r|r}
\hline \hline & $i$ & $c_{i}$ & $p_{i}$ & $q$ \\
\hline \hline$N_{\max }$ & 1 & 0.124 & 1.012 & 2.677 \\
& 2 & 0.244 & 0.902 & \\
\hline \hline & $i$ & $a_{i}\left(\mathrm{~g} / \mathrm{cm}^{2}\right)$ & $b_{i}\left(\mathrm{~g} / \mathrm{cm}^{2}\right)$ & $q$ \\
\hline \hline$X_{\max }$ & 1 & 366.2 & 139.5 & 3.117 \\
& 2 & 642.2 & 51.0 & \\
\hline$\lambda$ & 1 & 266.0 & 42.1 & 2.074 \\
& 2 & 398.8 & -21.9 & \\
\hline$X_{0}$ & 1 & -2.9 & -2.6 & 4.025 \\
& 2 & -15.8 & 0.6 & \\
\hline$f$ & 1 & 1 & 0.53 & 2.72 \\
& 2 & 2.45 & - & \\
\hline \hline Eq. (3): & \multicolumn{1}{|c|}{$K=12.4$} & $\alpha_{1}=0.787$ & $\alpha_{2}=5.99$ \\
\hline \hline
\end{tabular}

that the muon production peaks higher in the atmosphere for higher $E_{\mu}$. For this reason, it is best to optimize the parameterization using simulations for a specific energy range for the application at hand. In Table 3, we give a set of values fitted to simulations with a muon threshold of $50 \mathrm{GeV}$, which is used for calculations for NOvA Near Detector [19] in Section 4. For this case, also the behaviour of the $f$ ratio is different and it now has two regimes with a clear energy dependence instead of becoming constant at high $E_{0} / A E_{\mu}$. In Fig. 5, the muon multiplicities integrated over depth are compared with the standard Elbert formula for both energy regions.

A Python implementation of the parameterizations derived in this work can be found on GitHub².

\section{Surface-underground coincident measurements}

Before applying the parameterization in the context of underground muon rates, it is instructive to look at the case where the primary cosmic-ray energy is fixed, as can be achieved with coincident measurements of air showers with

\footnotetext{
${ }^{2}$ https://github.com/verpoest/muon-profile-parameterization
} 


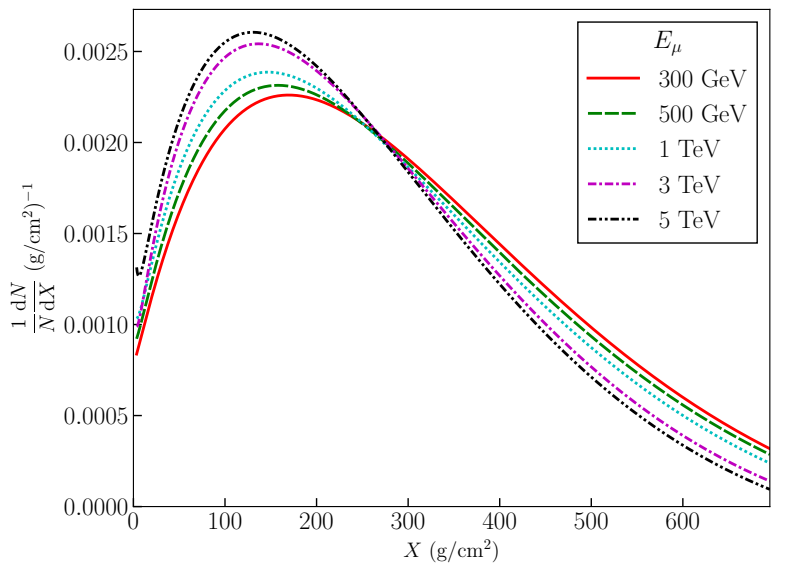

Figure 4: Normalized muon production profiles fit to vertical proton simulations with primary energies chosen so that $E_{0} / E_{\mu}=10^{4}$ for each threshold muon energy. There is a clear remaining $E_{\mu}$ dependence in the shapes, with production for higher energy muons peaking earlier in the atmosphere.
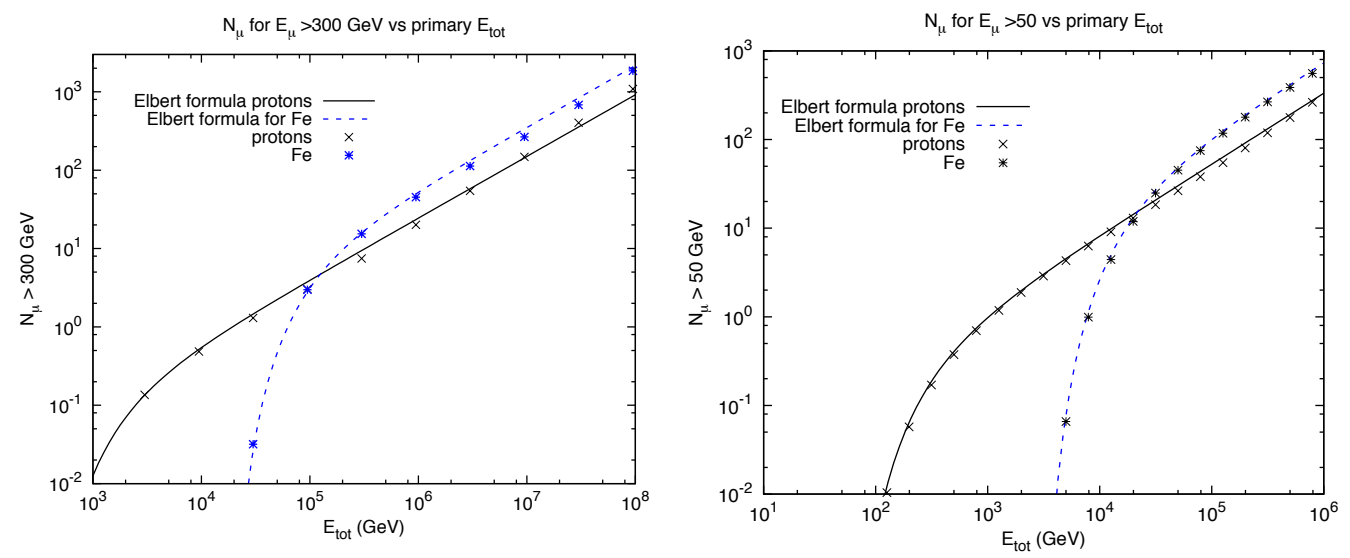

Figure 5: $N_{\mu}>E_{\mu, \text { min }}$ for vertical protons (solid) and Fe (dashed) from Eq. (3) compared to values from the integration of Eq. (4) for two energy regimes corresponding to Tables 1 and 3 . 
a surface array and an underground muon detector. Examples of experiments with this capacity are EAS-TOP and MACRO at Gran Sasso [20], the Baksan Underground Laboratory [21], SPASE-AMANDA [22, 23] and the IceTop and IceCube detectors at the South Pole [5]. We will use primary and muon energies relevant for the latter in our calculations.

As with the Elbert formula Eq. (3), it is possible to estimate the multiplicity of high-energy muons produced in a shower given a certain primary energy, mass and muon energy threshold by integrating over the production profile given by Eq. (4) and the parameterization of e.g. Table 1. More information about the muon bundle can however be extracted from the profiles, as the depth dependence of the muon production combined with a transverse momentum distribution allows one to estimate its lateral size. Furthermore, the dependence on the atmospheric temperature through the critical energy Eq. (5) for pions and kaons, enables the calculation of how both the muon bundle multiplicity and size vary throughout the year.

We estimate the seasonal variations of the multiplicity as relevant for IceCube, which measures muons with energies above approximately $400 \mathrm{GeV}$ from primary cosmic rays in the energy range $1 \mathrm{PeV}$ to $1 \mathrm{EeV}$. Atmospheric data is obtained from the AIRS satellite [24], which provides the temperature at different atmospheric pressure levels unevenly spaced between $1 \mathrm{hPa}$ and $700 \mathrm{hPa}$, between which we interpolate. Using the relation between vertical depth and pressure from the atmospheric overburden, the temperature as function of depth is obtained, which is used to calculate the muon production profiles. The resulting integral profiles can be seen for vertical showers of $10 \mathrm{PeV}$ primaries in Fig. 6a for three different days representing roughly the minimal, maximal and mean expected muon number in a year. During the austral summer, the atmosphere is warmer and less dense, causing more mesons to decay to high-energy muons instead of undergoing further interaction, and vice versa for the winter. This can clearly be seen in Fig. 6b, where the variation of the expected muon number throughout the year is shown for 5 different cosmic-ray mass groups. The maximal relative variations are of the order of $6 \%$ around the mean, slightly increasing with primary mass, and slowly decreasing with primary energy. This may be a relevant effect to take into account when deriving primary cosmic-ray composition from this observable by comparing experimental data to simulation. Variations of this kind have already been observed and were corrected for in Ref. [5, 25].

To estimate the transverse size of the muon bundle, the muon production as function of altitude rather than atmospheric depth is important, and needs 


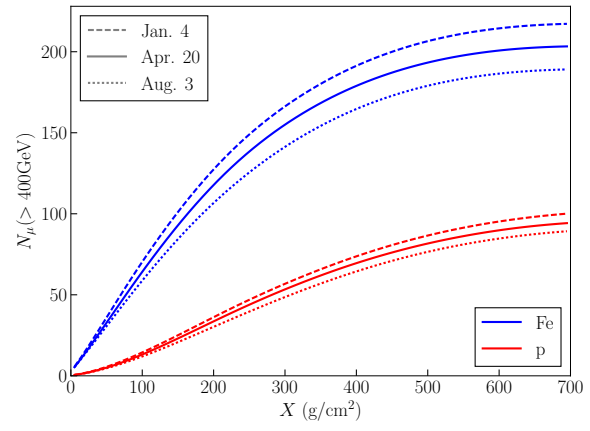

(a)

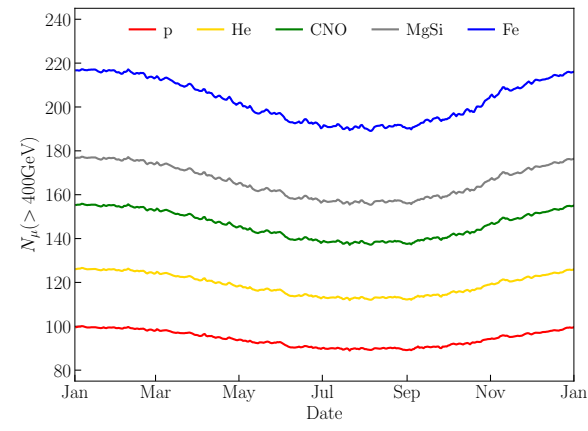

(b)

Figure 6: Effect of atmospheric variations on high-energy muon production in air showers. (a) Integral muon number throughout the atmosphere at the South Pole for vertical $10 \mathrm{PeV}$ proton and iron primaries for three different days of 2017, corresponding to days with approximately minimum, maximum and mean muon multiplicities. (b) Variation of the expected muon multiplicity for five different mass groups throughout the year.

to be combined with a transverse momentum distribution for the muons. The transverse distance from the shower axis of a muon with energy $E_{\mu}$ produced at an altitude $h$ is given by

$$
r_{T}=\frac{p_{T}}{E_{\mu}} \times \frac{h}{\cos \theta}
$$

where $\theta$ is the zenith angle of the primary and $p_{T}$ is the transverse momentum of the muon. The altitude corresponding to the vertical depth $X_{v}$ where the temperature is measured, is calculated using the ideal gas law, which gives

$$
h\left(X_{v}\right)=\frac{R T}{M g} \ln \frac{X_{0}}{X_{v}}
$$

with $X_{0}$ the vertical depth at $h=0$. A transverse momentum distribution $\mathrm{d} \sigma / \mathrm{d} p_{T}^{2}=f\left(p_{T}\right)$ can be assumed for the muons, relative to the direction of the incident primary particle, with the normalized distribution given by

$$
f\left(p_{T}\right)=\frac{4 p_{T}}{\left\langle p_{T}\right\rangle^{2}} e^{-2 p_{T} /\left\langle p_{T}\right\rangle}
$$

where $\left\langle p_{T}\right\rangle \approx 350 \mathrm{MeV}[26,27]$. We perform a simple estimate of the expected bundle size using this mean value of the transverse momentum for 
the surface above IceCube, located at an elevation of $2835 \mathrm{~m}$ with an atmospheric depth of about $700 \mathrm{~g} / \mathrm{cm}^{2}$, which is used as the zero point for our altitude calculations. Fig. 7a shows the muon production differential in depth as function of altitude for proton and iron primaries and at three dates again representing roughly an average and two extremal days. It is clear that muon production happens higher up in the atmosphere for heavy primaries. For a specific primary mass, muons are also produced higher in the atmosphere in summer compared to winter, because of its thermal expansion. Calculating the transverse distance for a muon with an average $p_{T}$ produced at a depth $X$ using Eq. (8), and taking the weighted average by multiplying with the production profile and integrating over depth, gives an estimate of the expected bundle radius for muons with energy $>E_{\mu}$, in this case $400 \mathrm{GeV}$. Results of this calculation for various primary masses over a full year are shown in Fig. 7b. The summer maximum caused by the change in production altitude can clearly be observed. The seasonal variations in the size are of the order of $10 \%$. One can also calculate the size for muons with the average muon energy in the shower instead of the minimum energy by multiplying $E_{\mu}$ in Eq. (8) by the $f$ ratio defined in the previous section.

A full calculation of the muon bundle properties in the detector would need to include the propagation to the detector, where multiple Coulomb scattering [28] in the overburden would further separate the muons, as well as charge separation of the muons caused by the geomagnetic field [29], but is beyond the scope of this example.

The simple calculation using the parameterization predicts a muon bundle size that is about $40 \%$ larger for iron primaries than for protons. The amount of muons expected for iron primaries is, on the other hand, roughly a factor of 2 higher than for protons. A similar conclusion was reached as a result of simulations in Ref. [10].

\section{Rates of muons in underground detectors}

The rate of muons of energy $>E_{\mu}$ from a direction corresponding to zenith angle $\theta$ in a detector with area $A_{\text {eff }}$ is given by

$$
I_{\mu}\left(>E_{\mu, \min }, \theta\right)=A_{\text {eff }}\left(E_{\mu, \min }, \theta\right) \int \mathrm{d} X \int_{E_{\mu, \min }, \theta} \mathrm{d} E_{\mu} P\left(E_{\mu}, \theta, X\right)
$$




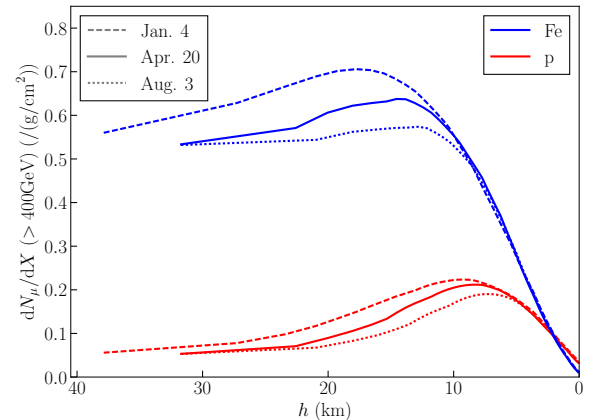

(a)

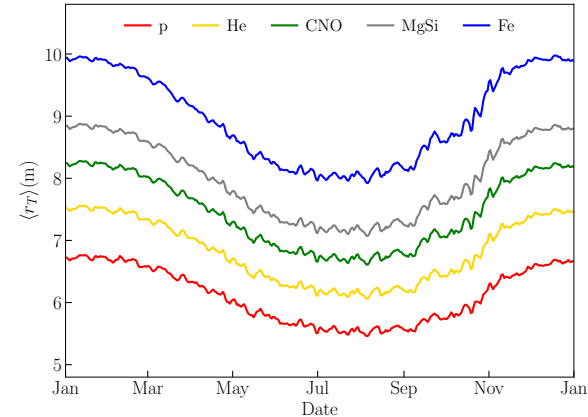

(b)

Figure 7: Effect of atmospheric variations on high-energy muon bundle size. (a) Muon production as function of height above the surface for $10 \mathrm{PeV}$ proton and iron primaries at the South Pole on three different days of 2017, corresponding to days with approximately minimum, maximum and mean muon multiplicities. (b) Variation of the mean bundle size for five different mass groups throughout the year.

where now

$$
P\left(E_{\mu}, \theta, X\right)=\int_{E_{\mu, \min }, \theta} \frac{\mathrm{d} N}{\mathrm{~d} X}\left(>E_{\mu, \min }, E_{0}, A=1, \theta, X\right) \phi\left(E_{0}\right) \mathrm{d} E_{0}
$$

is the production spectrum of muons differential in slant depth $X$ from Eq. (4) folded with the primary spectrum. We use the superposition approximation with $\phi\left(E_{0}\right)$ the spectrum of nucleons per $\mathrm{GeV} /$ nucleon summed over all nuclei using the H3A model [30] to estimate rates of events.

In this section, we consider three compact underground detectors, the MINOS Far Detector (FD) at Soudan [4], the MINOS Near Detector (ND) at Fermilab [31] and the NOvA ND [19], also at Fermilab. For these detectors, all muons with energy greater than the minimum to reach the detector can be counted and the same energy threshold applies to all muons in an event from a given direction. The MINOS FD is at a depth corresponding to a minimum muon energy of $730 \mathrm{GeV}$, which increases with zenith according to the energy-loss for each slant depth. In contrast, the shallow MINOS ND and the NOvA ND have thresholds around $50 \mathrm{GeV}$. In this case we account for muon decay and energy loss in the atmosphere, which reduces the rate by a few per cent. In both cases the rates are calculated and summed over 8 bins of zenith angles with threshold muon energies given in Table 2.

The temperature dependence of the muon production spectrum is entirely contained in the critical energies given in Eq. (5). The positive correlation 
Table 2: Minimum muon energies $(\mathrm{GeV})$ for 8 bins of $\cos \theta$

\begin{tabular}{r|c|c|c|c|c|c|c|c}
\hline $\cos \theta$ & 0.95 & 0.85 & 0.75 & 0.65 & 0.55 & 0.45 & 0.35 & 0.25 \\
\hline MINOS FD & 730 & 850 & 1030 & 1320 & 1800 & 2730 & 5000 & 14000 \\
\hline NOvA ND & 50 & 56 & 64 & 74 & 89 & 111 & 147 & 217 \\
\hline
\end{tabular}

of the overall rate with temperature reflects the higher probability of decay of the parent mesons to muons compared to re-interaction when the density is lower. Event rates are generally higher in summer and lower in winter. The correlation coefficient is larger for the deeper MINOS FD [4] than for the shallow detectors. This is because, at the lower energy threshold, pions still have a high probability of decaying, so the correlation of the charged pion decay channel is only weakly coupled to temperature. For quantitative estimates of correlation of rates with effective temperature in this section we use the temperature profiles from the AIRS satellite data [24] at the locations of Soudan and Fermilab to calculate $T_{\text {eff }}$. The effective temperature is evaluated as

$$
T_{\text {eff }}(\theta)=\frac{\int \mathrm{d} X P\left(E_{\mu}, \theta, X\right) T(X)}{\int \mathrm{d} X P\left(E_{\mu}, \theta, X\right)},
$$

with $P\left(E_{\mu}, \theta, X\right)$ defined in Eq. (12). ${ }^{3}$ In Eq. $13 A_{\text {eff }}\left(E_{\mu, \min }\right)$ factors out of the integrals and cancels in the ratio.

Both MINOS detectors [6] and the NOvA ND [19] report anti-correlation with $T_{\text {eff }}$ in the rates of events with two or more muons. This is in contrast with the seasonal variation of the total rate, which peaks in the summer when temperatures are higher. Our goal here is to use the parameterization to estimate the extent to which the anti-correlation for multiple muons can be accounted for by the effect of larger muon bundle radius in summer when muon production occurs higher in the atmosphere (see Fig. 7a).

The mean perpendicular distance from the shower axis of a muon produced at altitude $h$ with zenith angle $\theta$ is given by Eq. (8). To calculate the distance from the shower axis at the level of the detector, $r_{T}(X)$ is weighted with the production spectrum for each angular bin with slant depth $X$ related

\footnotetext{
${ }^{3} \mathrm{~A}$ different expression that uses the derivative with respect to temperature of the muon production spectrum [32] is used by MINOS and other underground detectors. We note the relatively small difference of using that definition in the analysis of the MINOS FD below.
} 
to altitude by Eq. (9).

$$
\left\langle r_{T}(\theta)\right\rangle=\frac{\int \mathrm{d} E_{\mu} \int d X r_{T}(X) P_{\mu}\left(E_{\mu}, \theta, X\right)}{\int \mathrm{d} E_{\mu} \int d X P_{\mu}\left(E_{\mu}, \theta, X\right)} .
$$

Finally, $\left\langle r_{T}(\theta)\right\rangle$ is integrated over zenith angle.

There are two other processes that affect the separation of muons from the shower axis and from each other: multiple scattering in the overburden and separation of muons by bending in the geomagnetic field before they reach the surface. Only the latter is important for the shallow detectors. We first analyse the altitude effect and then comment on multiple scattering and magnetic deviations.

\subsection{The MINOS Far Detector at Soudan}

The MINOS FD is at a depth of 2100 m.w.e., which corresponds to $E_{\mu, \text { min }} \approx 730 \mathrm{GeV}$. In this case, the parameters of Table 1 are appropriate. Integrating $r_{T}$ over production depth (altitude) and zenith angle, we find that the characteristic distance is comparable to the $8 \mathrm{~m}$ lateral dimension of the detector. For the assumed transverse momentum distribution of Eq. (10),

$$
\text { Fraction }=1-\left(1+\frac{2 p_{T}}{\left\langle p_{T}\right\rangle}\right) \times e^{-2 p_{T} /\left\langle p_{T}\right\rangle}
$$

gives the fraction of muons with momentum less than $p_{T}$. The convolution of this fraction ${ }^{4}$ with the muon production spectrum as a function of slant depth (altitude) is calculated for three values of $p_{T, i}$ at each depth $i$ corresponding to $r_{T}=8,4.5$ and $0.6 \mathrm{~m}$ at the ground. Eq. (10) is used to find $p_{T, i}$ at each depth $i$ corresponding to the three values of separation that characterize multiple muon events in the MINOS FD. In this way the partial rates for regions A $(0.6<r<4.5 \mathrm{~m}), \mathrm{B}(4.5<r<8 \mathrm{~m})$ and $\mathrm{C}(r>8 \mathrm{~m})$ are estimated. The idea for this simple interpretation is that in an event with two or more muons, the closest muon to the shower axis is a proxy for the center of the distribution, and the overall lateral distribution then provides an estimate of the distance to other muons. Approximating the MINOS FD as a cylinder of $8 \mathrm{~m}$ diameter and length $31 \mathrm{~m}$, the total rate is estimated as $\approx 0.4 \mathrm{~Hz}$. The Elbert formula (Eq. (3)) weighted by the primary spectrum and assuming

\footnotetext{
${ }^{4}$ Replace $r_{T}(X)$ in Eq. (14) with Fraction(X).
} 

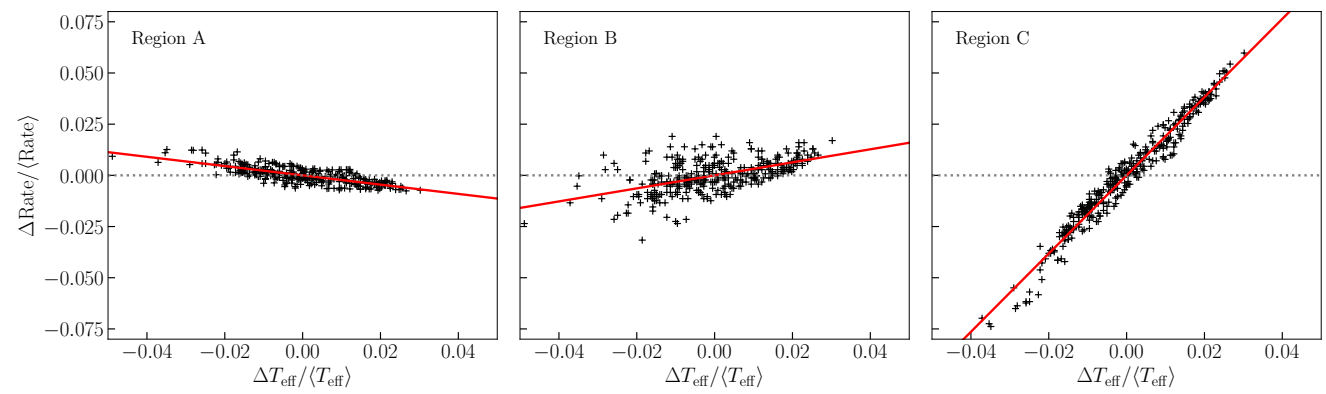

Figure 8: Correlation of Regions A, B, and C in the MINOS Far Detector with $T_{\text {eff }}$. Partial correlation coefficients from the altitude effect only are $-0.23,0.32$ and 1.91 respectively for $\mathrm{A}, \mathrm{B}$, and $\mathrm{C}$.

a Poisson multiplicity distribution is used to estimate the fraction of events with two or more muons at the depth of the detector as 0.072 . With these assumptions, the predicted rate of multiple events is $0.028 \mathrm{~Hz}$, a factor of two larger than observed. This discrepancy is in part due to the fact that the shape of the detector is not accounted for in calculating the partial rates. For example, containment for events with two muons separated by $<8 \mathrm{~m}$ would need to account for their orientation in azimuth relative to the long axis of the detector.

Our result is shown in Fig. 8 for calendar year 2009 by plots of the correlation between rates and effective temperature for the three regions. The effect of multiple scattering is to add about $\approx 2 \mathrm{~m}$ to the separation of muons for deep detectors [28]. The separation from propagation through the geomagnetic field [29] is $\approx 1.5 \mathrm{~m}$ for $\mathrm{TeV}$ muons. To estimate the effects of multiple scattering and magnetic deflection, we therefore reduce the radii for the atmospheric effect from $(0.6,4.5,8) \mathrm{m}$ to $(0.1,2.5,6$. $) \mathrm{m}$. Doing so increases the anti-correlation effect, changing the correlation coefficients for regions $(A, B, C)=(-0.23,0.32,1.91)$ to $(-0.46,0.05,1.54)$. Using a definition of effective temperature similar to that of Ref. [32] gives the values $(A, B, C)=(-0.51,0.09,1.69)$ for the calculation with the reduced atmospheric effect.

The MINOS FD paper does not give explicit values for the correlation coefficients for Regions A,B,C. However, these can be inferred from Table 1 of Ref. [6], which gives the amplitudes and phases of a sinusoidal fit to the rates in the three regions as well as corresponding values for their earlier measurement of single muons in the MINOS FD. The correlation coefficient 
for the single muons is 0.873 [4] and the amplitude of the sinusoidal fit for single muons is $1.27 \%$. The correlation coefficients inferred in this way from the amplitudes and phases of regions $\mathrm{A}$ and $\mathrm{C}$ are respectively -0.69 and +1.38 , with $\mathrm{B}$ intermediate. The trend from negative correlation in region $\mathrm{A}$ to strong positive correlation in Region $\mathrm{C}$ is present in our calculation, and motivates further investigation accounting in detail for all three separation effects as well as detector geometry.

Table 3: Parameter values for Eq. (7) for $>50 \mathrm{GeV}$ muons.

\begin{tabular}{|c|c|c|c|c|}
\hline & $i$ & $c_{i}$ & $p_{i}$ & $q$ \\
\hline \multirow[t]{3}{*}{$N_{\max }$} & 1 & 0.144 & 0.972 & \multirow[t]{2}{*}{2.557} \\
\hline & 2 & 0.213 & 0.905 & \\
\hline & $i$ & $a_{i}\left(\mathrm{~g} / \mathrm{cm}^{2}\right)$ & $b_{i}\left(\mathrm{~g} / \mathrm{cm}^{2}\right)$ & $q$ \\
\hline \multirow[t]{2}{*}{$X_{\max }$} & 1 & 260.9 & 176.4 & \multirow[t]{2}{*}{3.476} \\
\hline & 2 & 665.1 & 60.1 & \\
\hline \multirow[t]{2}{*}{$\lambda$} & 1 & 289.4 & 95.0 & \multirow[t]{2}{*}{1.526} \\
\hline & 2 & 483.0 & -31.8 & \\
\hline \multirow[t]{2}{*}{$X_{0}$} & 1 & -28.7 & -2.3 & \multirow[t]{2}{*}{2.778} \\
\hline & 2 & -48.0 & 4.6 & \\
\hline \multirow[t]{2}{*}{$f$} & 1 & 1 & 0.69 & \multirow[t]{2}{*}{2.80} \\
\hline & 2 & 3.06 & -0.05 & \\
\hline Eq. (3): & & $K=6.034$ & $\alpha_{1}=0.80$ & $\alpha_{2}=5.99$ \\
\hline
\end{tabular}

\subsection{Shallow detectors at Fermilab}

For the MINOS and the NOvA ND, both at $\approx 200$ m.w.e., where $E_{\mu, \min } \sim$ $50 \mathrm{GeV}$, we use parameters of Table 3, tuned for the lower energy region. The main practical difference at the lower energy is that the muon bundle size of $\approx 60 \mathrm{~m}$ is much larger than the scale of the detector. In this case, the rate of multiple muon events is determined primarily by the small probability of a second muon to find the detector, as well as by the fraction of events with two or more muons at the depth. Accordingly, we estimate the fraction of events with multiple muons by converting the transverse momentum variables in Eq. (15) to lateral distances from the shower axis using the weighted distribution of altitude $/ \cos \theta$. For a simple estimate we calculate the probability 

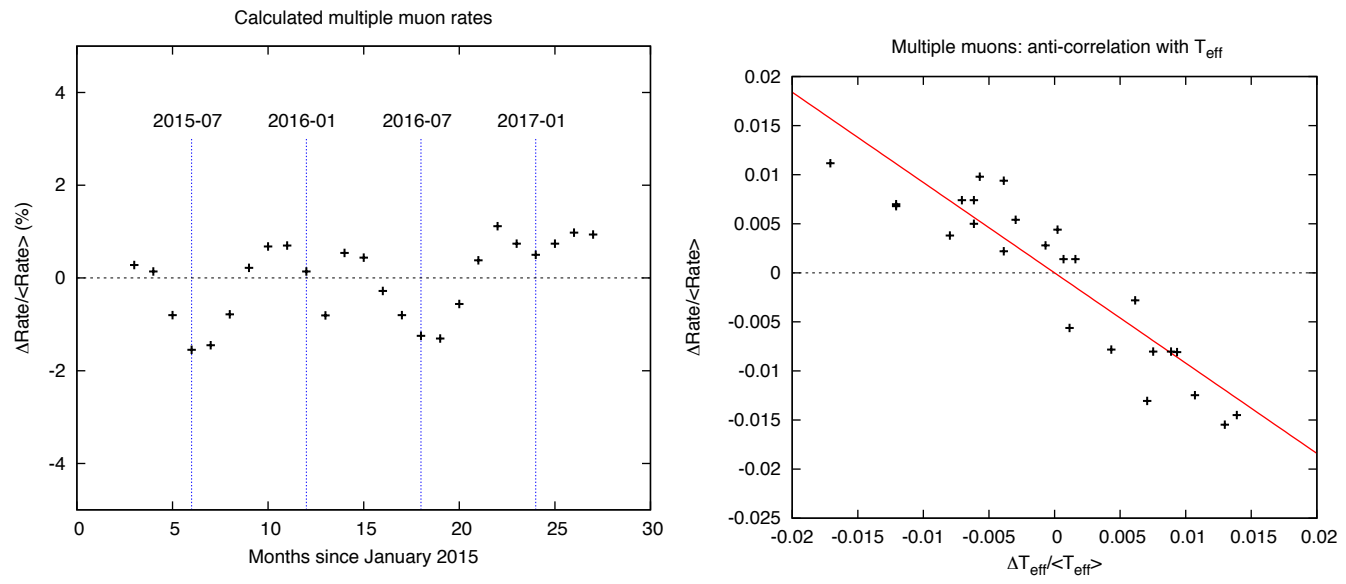

Figure 9: Calculated rates (left) and correlation (right) for multiple muon events at NOvA.

that a second muon lies within $7 \mathrm{~m}$ of the shower axis. We find that $3.3 \%$ of muons satisfy this condition. Our calculated total rate in the NOvA ND with $E_{\mu, \min }=50 \mathrm{GeV}$ is $50 \mathrm{~Hz}$. Using the Elbert formula Eq. (3) with the assumption of a Poisson distribution, we estimate that $16 \%$ of events have two or more muons at the depth of NOvA. The product $\approx 0.26 \mathrm{~Hz}$ is somewhat greater than the observed rate of multiple muon events in Ref. [19], which is $0.15 \mathrm{~Hz}$.

Results of our calculations for multiple muons at NOvA are shown in Fig. 9. The monthly averages show the anti-correlation with effective temperature as in Figs. 3 and 4 of Ref. [19], but the amplitude of the variation is smaller. The right panel of Fig. 9 shows a correlation coefficient of $\sim-0.92$, as compared to -4.14 reported in Ref. [19]. The separation from propagation in the geomagnetic field for $\sim 50 \mathrm{GeV}$ muons at Fermilab is only somewhat less than the $60 \mathrm{~m}$ scale of the altitude effect. To estimate the geomagnetic effect, we reduce the parameter of the altitude effect from 7 to $4 \mathrm{~m}$. This gives only a small increase in the anti-correlation effect, changing the correlation coefficient from -0.92 to -1.00 . In summary, the analysis described here does not fully explain the observed anti-correlation in NOvA, indicating that a full simulation is required. 


\section{Summary}

The main point of this paper is to provide a parameterization of the muon production profile as a function of atmospheric depth and zenith angle for primary protons and nuclei. The parameters of muon production versus slant depth in the atmosphere (Eq. (4)) are given for two regions of muon energy, $\sim \mathrm{TeV}$ and $\sim 100 \mathrm{GeV}$ in Tables 1 and 3 . The production profile can be folded with atmospheric temperature profiles to obtain the multiplicity and size of muon bundles in air showers for which the primary energy is determined by a surface array (Section 3). Future application of the parameterization of Table 1 to seasonal variations of muon events in IceCube requires implementation of the formulas in a framework that accounts separately for the contribution from each bin of muon energy at each depth to be folded with an effective area function that depends both on direction and energy.

The production profiles can also be used as a weighting factor to calculate effective temperatures for analysis of seasonal variations in underground detectors, including events with multiple muons (Section 4). Our focus in the latter application is to investigate the altitude effect by which parent mesons decay higher in the summer causing multiple muon events to be more spread out at the detector. We find that this effect makes an important contribution to the observed anti-correlation with temperature of multiple muon events for both the MINOS FD at TeV energies and for the shallow detectors at lower energy. With the muons more spread out, the rate at small separations decreases.

For the MINOS FD [6] the size of the TeV muon bundles is comparable to the detector area. In this case we find that the altitude effect reverses the correlation coefficient for the innermost selection region, and that the anticorrelation becomes more prominent when the additional effects of multiple scattering in the overburden and bending in the geomagnetic field are accounted for. At the shallow detectors at Fermilab with lower energy muons, the multiple muon events have a characteristic size much larger than the area of the detector and only the geomagnetic effect is significant. For the NOvA ND we find an anti-correlation for all multiple muon events but with an amplitude significantly smaller than observed. Our approximate results indicate the need in both cases for more extensive analyses that account for the details of detector acceptance and for the additional effects of multiple scattering and charge separation in the geomagnetic field.

Acknowledgments: We thank Spencer Klein for reading an initial draft of 
the paper and pointing out the importance of multiple scattering and bending in the geomagnetic field for the analysis of multiple muon events. We thank the referee who also noted the relevance of multiple scattering. We thank Dennis Soldin, Segev BenZvi and Carlos Argüelles for helpful comments on this work. S.V. acknowledges the Fund for Scientific ResearchFlanders (FWO) and the FWO Big Science programme. S.V. appreciates the hospitality of the Bartol Research Institute, where the idea for this paper emerged.

\section{References}

[1] P. Barrett, et al., Interpretation of cosmic-ray measurements far underground, Rev. Mod. Phys. 24 (1952) 133-178.

[2] P. Desiati, T. Kuwabara, T. K. Gaisser, S. Tilav, D. Rocco, Seasonal Variations of High Energy Cosmic Ray Muons Observed by the IceCube Observatory as a Probe of Kaon/Pion Ratio, in: Proceedings, 32nd International Cosmic Ray Conference (ICRC 2011): Beijing, China, August 11-18, 2011, vol. 1, 78-81, doi:10.7529/ICRC2011/ V01/0662, 2011.

[3] S. Tilav, T. K. Gaisser, D. Soldin, P. Desiati, Seasonal variation of atmospheric muons in IceCube, PoS ICRC2019 (2020) 894, doi:10.22323/1.358.0894.

[4] P. Adamson, et al., Observation of muon intensity variations by season with the MINOS far detector, Phys. Rev. D81 (2010) 012001, doi:10.1103/PhysRevD.81.012001.

[5] M. Aartsen, et al., Cosmic ray spectrum and composition from $\mathrm{PeV}$ to $\mathrm{EeV}$ using 3 years of data from IceTop and IceCube, Phys. Rev. D 100 (8) (2019) 082002, doi: 10.1103/PhysRevD.100.082002.

[6] P. Adamson, et al., Observation of Seasonal Variation of Atmospheric Multiple-Muon Events in the MINOS Near and Far Detectors, Phys. Rev. D91 (11) (2015) 112006, doi:10.1103/PhysRevD.91.112006.

[7] J. W. Elbert, Multiple muons produced by cosmic ray interactions., in: Proceedings of the DUMAND Summer Workshop, Scripps Institution of Oceanography, La Jolla CA, 101-121, 1979.

[8] J. W. Elbert, Cosmic ray multiple muon events in deep detectors., in: 16th International Cosmic Ray Conference. Vol. 10. Conference Papers. Mn Session. Proceedings, Kyoto, Japan, 6-18 August 1979, 405-409, 1979.

[9] T. Gaisser, T. Stanev, Muon bundles in underground detectors, Nucl. Instrum. Meth. A 235 (1985) 183-192, doi:10.1016/0168-9002(85)90260-8. 
[10] C. Forti, H. Bilokon, B. d'Ettore Piazzoli, T. Gaisser, L. Satta, T. Stanev, Simulation of atmospheric cascades and deep underground muons, Phys. Rev. D 42 (1990) 36683689, doi:10.1103/PhysRevD.42.3668.

[11] T. K. Gaisser, R. Engel, E. Resconi, Cosmic Rays and Particle Physics, Cambridge University Press, ISBN 9780521016469, 9781316598917, 2016.

[12] T. Gaisser, A. Hillas, Reliability of the method of constant intensity cuts for reconstructing the average development of vertical showers, Proceedings, 15th International Cosmic Ray Conference (ICRC1977): Plovdiv, Bulgaria 8 (1977) 353-357.

[13] D. Heck, J. Knapp, J. N. Capdevielle, G. Schatz, and T. Thouw, CORSIKA: A Monte Carlo code to simulate extensive air showers .

[14] F. Riehn, R. Engel, A. Fedynitch, T. K. Gaisser, T. Stanev, The hadronic interaction model Sibyll 2.3c and extensive air showers .

[15] S. A. Bass, et al., Microscopic models for ultrarelativistic heavy ion collisions, Prog. Part. Nucl. Phys. 41 (1998) 255-369, doi:10.1016/S0146-6410(98)00058-1.

[16] M. Bleicher, et al., Relativistic hadron hadron collisions in the ultrarelativistic quantum molecular dynamics model, J. Phys. G 25 (1999) 1859-1896, doi: 10.1088/0954-3899/25/9/308.

[17] De Ridder, Sam, Sensitivity of IceCube cosmic ray measurements to the hadronic interaction models, Ph.D. thesis, Ghent University, 2019.

[18] C. Song, Longitudinal profile of extensive air showers, Astropart. Phys. 22 (2004) 151-158, doi:10.1016/j.astropartphys.2004.05.011.

[19] M. A. Acero, et al., Observation of seasonal variation of atmospheric multiple-muon events in the NOvA Near Detector, Phys. Rev. D99 (12) (2019) 122004, doi:10.1103/ PhysRevD.99.122004.

[20] M. Aglietta, et al., The cosmic ray proton, helium and CNO fluxes in the 100$\mathrm{TeV}$ energy region from $\mathrm{TeV}$ muons and EAS atmospheric Cherenkov light observations of MACRO and EAS-TOP, Astropart. Phys. 21 (2004) 223-240, doi: 10.1016/j.astropartphys.2004.01.005.

[21] V. Bakatanov, Y. Novoseltsev, R. Novoseltseva, Observation of 'the knee' in cosmic ray energy spectrum with underground muons and primary mass composition in the range $10^{* *} 15-\mathrm{eV}$ to $10^{* *} 17-\mathrm{eV}$, Astropart. Phys. 12 (1999) 19-24, doi:10.1016/ S0927-6505(99)00074-2.

[22] J. Ahrens, et al., Calibration and survey of AMANDA with the SPASE detectors, Nucl. Instrum. Meth. A 522 (2004) 347-359, doi:10.1016/j.nima.2003.12.007. 
[23] J. Ahrens, et al., Measurement of the cosmic ray composition at the knee with the SPASE-2/AMANDA-B10 detectors, Astropart. Phys. 21 (2004) 565-581, doi: 10.1016/j.astropartphys.2004.04.007.

[24] NASA-AIRS, https://airs.jpl.nasa.gov/data/get-data .

[25] S. De Ridder, T. Feusels, Seasonal variation of the muon multiplicity in cosmic rays at South Pole, in: 33rd International Cosmic Ray Conference, 0763, 2013.

[26] B. Alper, et al., Large angle inclusive production of charged pions at the cern isr with transverse momenta less than 1.0 gev/c, Phys. Lett. B 47 (1973) 75-79, doi: 10.1016/0370-2693(73)90573-X.

[27] B. Alper, et al., Large angle inclusive production of protons, anti-protons and kaons, and particle composition at the cern isr, Phys. Lett. B 47 (1973) 275-280, doi:10. 1016/0370-2693(73)90730-2.

[28] P. Lipari, T. Stanev, Propagation of multi - TeV muons, Phys. Rev. D 44 (1991) 3543-3554, doi:10.1103/PhysRevD.44.3543.

[29] P. Abreu, et al., The effect of the geomagnetic field on cosmic ray energy estimates and large scale anisotropy searches on data from the Pierre Auger Observatory, JCAP 11 (2011) 022, doi:10.1088/1475-7516/2011/11/022.

[30] T. K. Gaisser, Spectrum of cosmic-ray nucleons, kaon production, and the atmospheric muon charge ratio, Astropart. Phys. 35 (2012) 801-806, doi:10.1016/j. astropartphys.2012.02.010.

[31] P. Adamson, et al., Observation of muon intensity variations by season with the MINOS Near Detector, Phys. Rev. D90 (1) (2014) 012010, doi:10.1103/PhysRevD. 90.012010 .

[32] E. Grashorn, J. de Jong, M. Goodman, A. Habig, M. Marshak, S. Mufson, S. Osprey, P. Schreiner, The Atmospheric charged kaon/pion ratio using seasonal variation methods, Astropart. Phys. 33 (2010) 140-145, doi:10.1016/j.astropartphys.2009.12.006. 